\title{
An Exhaustive Criterion for the Non-Existence of Invariant Circles for Area-Preserving Twist Maps
}

\section{J. Stark *}

Mathematics Institute, University of Warwick, Coventry CV4 7AL, United Kingdom

\begin{abstract}
We present a necessary and sufficient condition for the non-existence of rotational invariant circles for area-preserving twist maps of the cyclinder or annulus based on the "cone-crossing" and "killends" criteria of MacKay and Percival (1985). Given a number of technical restrictions on the implementation of these criteria, this condition leads to a proof of MacKay and Percival's Finite Computation Conjecture.
\end{abstract}

\section{Introduction}

Much interest has recently been focused on criteria for the non-existence of rotational invariant circles for area-preserving twist maps of the cylinder or annulus [(Chirikov, 1979; Greene, 1979; Mather, 1982, 1984; Newman and Percival, 1983; Aubry, 1983; Katok, 1983; Boyland and Hall, 1985), etc.]. MacKay and Percival (1985) unify a number of related criteria and present a practical algorithm which can be rigorously implemented on a digital computer. Herman (1983) uses essentially the same criterion in his construction of $C^{2-\varepsilon}$ counterexamples to the Moser Twist Theorem whilst Mather (1984) and Aubry (1983) apply this criterion to the standard map:

$$
\begin{aligned}
& r^{\prime}=r-(k / 2 \pi) \sin 2 \pi \theta, \\
& \theta^{\prime}=\theta+r^{\prime}
\end{aligned}
$$

to show that there are no rotational invariant circles for respectively $|k|>4 / 3$ and $|k|>\beta$, where $\beta \simeq 1.23 \ldots$ is the root of some transcendental equation. Aubry and Mather's work was done analytically, but by implementing the criterion on a computer MacKay and Percival are able to obtain a considerably better rigorous bound: there are no rotational invariant circles for $|k| \geqq 63 / 64=0.984375$. This is

\footnotetext{
* Present address: Department of Mathematics, Imperial College, 180 Queen's Gate, London SW7 2BZ, United Kingdom
} 
probably close to optimal since the numerical work of Greene (1979) (and MacKay, 1982, 1983) strongly suggests that there exists a $k_{c} \simeq 0.971635 \ldots$ such that there are no rotational invariant circles for $|k|>k_{c}$, but there is at least one such circle for $|k| \leqq k_{c}$.

This result of MacKay and Percival is considerably better than that obtainable by any other known non-existence criterion. Thus for instance consider the simplest such possible criterion: if there is an orbit which "crosses" the annulus $\mathbb{T}^{1} \times[a, b] \subset \mathbb{T}^{1} \times \mathbb{R} \quad$ [i.e. there exists $x \in \mathbb{T}^{1} \times(-\infty, a)$ such that $f^{k}(x) \in \mathbb{T}^{1} \times(-\infty, b)$ for some $\left.k \in \mathbb{Z}\right]$ then there can be no rotational invariant circle contained in the annulus $\mathbb{T}^{1} \times[a, b]$. According to MacKay et al. (1985) and Chirikov (1979) the mean time to cross the "golden cantorus" for the standard map at $k=1.0$ is of the order of $10^{6}$. To prove non-existence of rotational invariant circles at this value of $k$ we would have to rigorously find such an orbit which is hardly feasible. It thus seems that this simple criterion is unlikely to succeed for even $k=1.0$. By comparison, the longest orbit segment considered by MacKay and Percival had length 96 . Their program took less than 90 min of CPU time on a NAS 9080 computer, which is not a particularly fast machine by today's standards. If more computer time was available their criterion would undoubtedly give even sharper results. Similarly Leage and MacKay (1986) conclude that in practice Boyland and Hall's "badly ordered periodic orbits" criterion (Boyland and Hall, $1985)$ is not very efficient.

It is then natural to ask whether this criterion is exhaustive, that is, given a point through which no rotational invariant circles pass, will the criterion succeed in proving so. This is essentially the same question as the following conjecture made by MacKay and Percival (1985).

Finite Computation Conjecture given a compact region through which no rotational invariant circles pass, then the algorithm will prove so using a finite amount of computation.

The purpose of this paper is to prove a theorem which implies this conjecture as long as one places a number of natural restrictions on the way in which the criterion is implemented (e.g. on the accuracy and order of computation etc.). These restrictions and the detailed proof of MacKay and Percival's conjecture can be found in Stark (1986). Briefly this theorem can be described as follows: the MacKay and Percival non-existence criterion is based on Birkhoff's theorem, which states that every rotational invariant circle of an area-preserving twist map is the graph of a Lipschitz function. Their algorithm proceeds by obtaining a sequence $K_{n}$ of increasingly sharp local Lipschitz constants which any rotational invariant circle would have to satisfy and then testing whether or not there are any curves at all (not necessarily invariant) which satisfy these Lipschitz constants. If not, then clearly there are no rotational invariant circles either. The theorem we prove (4.4) is the converse of this:

Theorem. Given an area-preserving twist map with zero net flux, then if $x$ is a point such that for all $n \in \mathbb{N}$ there is a curve through $x$ satisfying $K_{n}$, then there is an invariant circle through $x$. 


\section{The Cone-Crossing Criterion}

2.1. Definition. An area-preserving twist map of the cylinder $M=\mathbb{T}^{1} \times \mathbb{R}$ is an area, orientation and end preserving $C^{1}$ diffeomorphism $f: M \rightarrow M$ which satisfies $\partial \theta^{\prime} / \partial y \geqq K>0$, where $f(\theta, y)=\left(\theta^{\prime}, y^{\prime}\right)$. One often also considers twist maps of the annulus $\mathbb{T}^{1} \times[0,1]$. Everything below remains valid in this case as long as appropriate care is taken at the boundaries of the annulus.

We often prefer to work in the universal cover $\mathbb{R}^{2}$ of $\mathbb{T}^{1} \times \mathbb{R}$, and so define a lift to $\mathbb{R}^{2}$ of $f$ to be a diffeomorphism $F: \mathbb{R}^{2} \rightarrow \mathbb{R}^{2}$ such that $\pi \circ F=f \circ \pi$, where $\pi$ is the covering map $\pi(t, y)=((t \bmod 1), y)$. Note that if $R: \mathbb{R}^{2} \rightarrow \mathbb{R}^{2}$ is the translation $R(t, y)$ $=(t-1, y)$ then $F$ commutes with $R$, and if $F^{\prime}$ is another lift of $F$, then $F^{\prime}=F \circ R^{k}$ for some $k \in \mathbb{Z}$.

2.2. Definition. An invariant circle for an area-preserving twist map $f$ is a homeomorphic image of $\mathbb{T}^{1}$ which is invariant under $f$. A rotational invariant circle is one which is homotopically non-trivial, i.e. which is homotopic to the generator of the fundamental group of $\mathbb{T}^{1} \times \mathbb{R}$. Intuitively such a circle winds once around the cylinder.

MacKay and Percival's cone crossing criterion is based on the following theorem which was proved by Birkhoff in the 1920's. See Herman (1983) for a modern proof.

2.3. Theorem (Birkhoff). Every rotational invariant circle $\Gamma$ is the graph $\left\{(\theta, \gamma(\theta)): \theta \in \mathbb{T}^{1}\right\}$ of a Lipschitz function $\gamma: \mathbb{T}^{1} \rightarrow \mathbb{R}$.

Recall that $\gamma$ is Lipschitz if there exist constants $C^{+}, C^{-} \in \mathbb{R}$ such that $\forall \theta \theta^{\prime} \in \mathbb{T}^{1}$, (Fig. 1):

$$
C^{-} \leqq \frac{\gamma(\theta)-\gamma\left(\theta^{\prime}\right)}{\theta-\theta^{\prime}} \leqq C^{+} .
$$

We shall want to generalise this definition to allow $C^{ \pm}$to vary with position, and hence define:

2.4. Definition. A conefield $K$ consists of two functions $K^{-}, K^{+}: M \rightarrow \mathbb{R}$ (not necessarily continuous). A function $\gamma: \mathbb{T}^{1} \rightarrow \mathbb{R}$ is said to satisfy a conefield $K$ if

$$
-1 / 4 \leqq K^{-}(\theta, \gamma(\theta)) \leqq K^{+}(\theta, \gamma(\theta)) \leqq 1 / 4 \quad \forall \theta \in \mathbb{T}^{1}
$$

Fig. 1

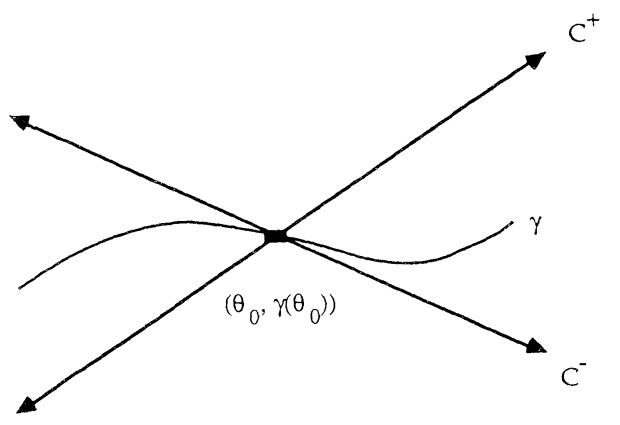


and $\tan \left(2 \pi K^{-}\right)$and $\tan \left(2 \pi K^{+}\right)$give local Lipschitz constants for $\gamma$ :

$$
\begin{aligned}
\tan 2 \pi K^{-}(\theta, \gamma(\theta)) & \leqq \liminf _{\theta \rightarrow \theta_{0}} \frac{\gamma(\theta)-\gamma\left(\theta_{0}\right)}{\theta-\theta_{0}} \forall \theta \in \mathbb{T}^{1}, \\
\limsup _{\theta \rightarrow \theta_{0}} \frac{\gamma(\theta)-\gamma\left(\theta_{0}\right)}{\theta-\theta_{0}} & \leqq \tan 2 \pi K^{+}(\theta, \gamma(\theta)) \forall \theta \in \mathbb{T}^{1} .
\end{aligned}
$$

Thus for instance if the graph of $\gamma$ is a $C^{1}$ curve, then the slope of the tangent vector to the curve at $x$ has to lie between $\tan \left(2 \pi K^{+}(x)\right)$ and $\tan \left(2 \pi K^{-}(x)\right)$. We shall say that a rotational invariant circle $\Gamma$ satisfies a conefield $K$ if the function whose graph $\Gamma$ is satisfies $K$.

The idea behind the cone-crossing criterion is to obtain a sequence $K_{m}$ of increasingly sharp conefields which any invariant circle has to satisfy. Then if for some $m \geqq 0$ and $x \in M$ we have $K_{m}^{+}(x)<K_{m}^{-}(x)$, there can be no rotational invariant circle through the point $x$.

By Birkhoff's theorem the constant conefield $K^{+}=+1 / 4, K^{--}=-1 / 4$ given by the upward and downward verticals is satisfied by all rotational invariant circles. We can then obtain further conefields from this one by using the action of the derivative $D f$. To define this action observe that we can think of a $K^{+}$and $K^{-}$as two sections of the line bundle $M \times \mathbb{R}$ considered as a cover of the unit tangent bundle $M \times \mathbb{T}^{1} \quad$ [with covering map $p: M \times \mathbb{R} \rightarrow M \times \mathbb{T}^{1}$ given by $p(x, t)$ $=(x,(t \bmod 1))]$. We take coordinates on $M \times \mathbb{T}^{1}$ so that $(x, \xi) \in M \times \mathbb{T}^{1}$ corresponds to the unit tangent vector $(\cos 2 \pi \xi, \sin 2 \pi \xi)$ at $x \in M$. We then lift these to $M \times \mathbb{R}$, thus $(x,+1 / 4)$ is the upward vertical at $x$ and $(x,-1 / 4)$ is the downward vertical at $x$. Then define:

2.5. Definition. The action of $D f$ on the tangent bundle of $M$ restricts to an action on the unit tangent bundle $M \times \mathbb{T}^{1}$. We can then lift this to an action on $M \times \mathbb{R}$, and since $D f$ is continuous and $f$ satisfies the twist property, there exists a unique continuous lift (which we continue to denote $D f$ ) such that $-1 / 4<D f_{x}(1 / 4)<1 / 4$, $\forall x \in M$. This action on $M \times \mathbb{R}$ then induces an operator on conefields (which we still denote by $D f$, Fig. 2) by

$$
\left[D f\left(K^{ \pm}\right)\right](x)=D f_{f^{-1}(x)}\left[K^{ \pm}\left(f^{-1}(x)\right)\right] .
$$

Similarly we get an action of $D f^{-1}$ by

$$
\left[D f^{-1}\left(K^{ \pm}\right)\right](x)=D f_{f(x)}^{-1}\left[K^{ \pm}(f(x))\right] .
$$

2.6. Definition. Define the conefields $K_{m}, m=0,1,2, \ldots$ by

$$
\begin{array}{ll}
K_{0}^{+}(x)=+1 / 4 & \text { (upward vertical) } \forall x \in M, \\
K_{0}^{-}(x)=-1 / 4 & \text { (downward vertical) } \forall x \in M,
\end{array}
$$

and

$$
\begin{aligned}
& K_{m}^{+}=D f\left(K_{m-1}^{+}\right) \forall m>0, \\
& K_{m}^{-}=D f^{-1}\left(K_{m-1}^{-}\right) \forall m>0 .
\end{aligned}
$$

2.7. Lemma. Any rotational invariant circle satisfies $K_{m}$ for all $m \geqq 0$. 


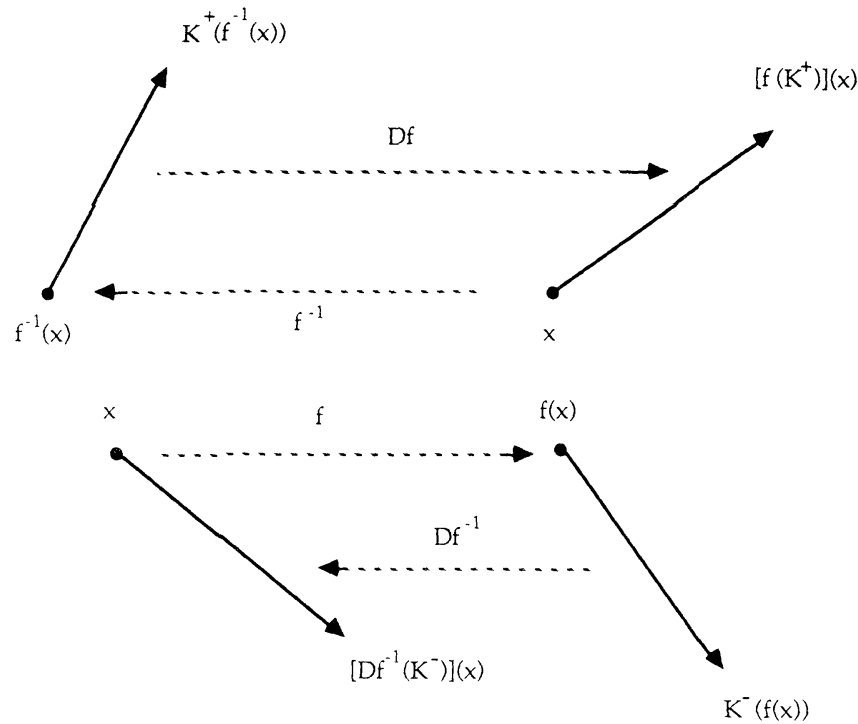

Fig. 2

$K^{-}(f(x))$

Proof. By induction. By Birkhoff's theorem all rotational invariant circles satisfy $K_{0}$. Now suppose that $\Gamma$ satisfies $K_{m-1}$ for some $m>0$. If for some $x \in \Gamma, K_{m}^{+}(x)$ is not a Lipschitz constant for $\Gamma$, then by definition $K_{m-1}^{+}\left(f^{-1}(x)\right)$ is not a Lipschitz constant for $f^{-1} \Gamma$ at $f^{-1}(x)$. But $\Gamma$ is $f$-invariant and hence $f^{-1} \Gamma=\Gamma$. Thus $K_{m-1}^{+}\left(f^{-1}(x)\right)$ is not a Lipschitz constant for $\Gamma$ at $f^{-1}(x)$ which contradicts the hypothesis that $\Gamma$ satisfies $K_{m-1}$. Similarly if $K_{m}^{-}(x)$ is not a Lipschitz constant for $\Gamma$ at $x$ then $K_{m-1}^{-}(f(x))$ is not a Lipschitz constant for $\Gamma$ at $f(x)$. Hence $\Gamma$ satisfies $K_{m}$

2.8. Cone-Crossing Criterion. If for some point $x \in M$ and some $m \in \mathbb{N}$ we have $K_{m}^{+}(x)<K_{m}^{-}(x)$, then there is no rotational invariant circle through $x$. We shall say that the "cones have crossed at $x$ " when this occurs.

2.9. Remark. By the twist condition we have $K_{1}^{+}(x)<1 / 4$ and $K_{1}^{-}(x)>-1 / 4$, $\forall x \in M$. Since the actions of $D f$ and $D f^{-1}$ on slopes are order preserving we get $K_{m}^{+}(x)<K_{m-1}^{+}(x)$ and $K_{m}^{-}(x)>K_{m-1}^{-}(x), \forall m \in \mathbb{N}, \forall x \in M$ (Fig. 3). Thus the action of $D f$ and $D f^{-1}$ gives us a sequence of increasingly sharp conefields.

2.10. Remark. All of the above concepts lift in a straightforward manner to the universal cover $\mathbb{R}^{2}$. Thus rotational invariant circles lift to graphs of periodic Lipschitz functions $\gamma: \mathbb{R} \rightarrow \mathbb{R}, \gamma(t+1)=\gamma(t), \forall t \in \mathbb{R}$. Conefields lift to functions $K^{-}, K^{+}: \mathbb{R}^{2} \rightarrow \mathbb{R}$ and we get an action of $D F$ and $D F^{-1}$ on conefields. Abusing notation, we shall continue to refer to the lift of the conefields $K_{m}$ as $K_{m}$.

2.11. Remark. In practice it is not feasible to perform the iteration (2.7) exactly, especially if one is using a digital computer. However one can obviously replace (2.7) by

$$
\begin{array}{ll}
K_{m}^{+}=\text {u.b. } D f\left(K_{m-1}^{+}\right) & \forall m>0, \\
K_{m}^{-}=1 . b . D f^{-1}\left(K_{m-1}^{-}\right) & \forall m>0,
\end{array}
$$


Fig. 3

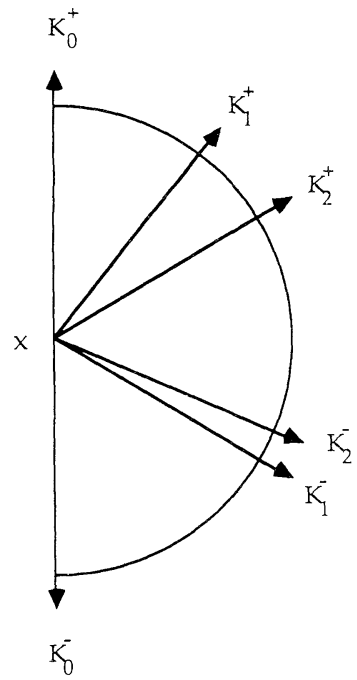

where u.b. is any upper bound and l.b. is any lower bound. If we restrict the $K_{m}$ to some class of functions which are representable in a finite manner (e.g. piece-wise constant functions) then we can easily perform (2.12) rigorously on a digital computer.

\section{Supplementary Criteria}

3.1. Remark. As MacKay and Percival (1985) show, the cones $K_{m}^{ \pm}$will never cross on an orbit of minimal action. On the other hand the work of Mather (1982) and Aubry and LeDaeron (1983) [see also MacKay and Stark (1985) for a survey] shows that typically there exist many orbits with minimal action which do not lie on rotational invariant circles. Thus just because the cones do not cross at a point does not imply that there is a rotational invariant circle through that point. In other words cone-crossing on its own is not an exhaustive criterion for the nonexistence of invariant circles. MacKay and Percival thus introduce a supplementary criterion which they call "killends." This is based on the observation that if for a given $x \in M$ all the curves through $x$ that satisfy some $K_{m}$ are forced to pass through a region where $K_{m}^{+}<K_{m}^{-}$, then there can be no rotational invariant circle through $x$. The "end" of the curve is "killed" even though at the starting point $x$ of the curve one might still have $K_{m}^{+}(x) \geqq K_{m}^{-}(x)$. More precisely define:

3.2. Definition. Given $x \in M$, choose $z \in \mathbb{R}^{2}$ such that $\pi(z)=x$, where $\pi$ is the covering $\operatorname{map} \pi(t, y)=((t \bmod 1), y)$. For $m>0$ define:

$$
\begin{aligned}
& L_{z}^{*}\left(K_{m}\right)=\left\{\gamma: \mathbb{R} \rightarrow \mathbb{R} \text { such that } z \in \text { graph } \gamma, \gamma \text { satisfies } K_{m}\right\}, \\
& L_{z}\left(K_{m}\right)=\left\{\gamma \in L_{z}^{*} \text { such that } \gamma(t+1)=\gamma(t), \forall t \in \mathbb{R}\right\} .
\end{aligned}
$$

Note that $\gamma$ is not required to be $f$-invariant. The set $L_{z}\left(K_{m}\right)$ thus consists of those closed curves $\gamma$ through $z$ that satisfy the conefield $K_{m}$. Observe that if $\gamma \in L_{z}\left(K_{m}\right)$ then $f^{-i}(\gamma)$ and $f^{i}(\gamma)$ will satisfy $K_{0}$ for all $1 \leqq i \leqq m$. We can thus characterise 
$L_{z}\left(K_{m}\right)$ as the set of all closed curves $\gamma$ through $z$ such that for all $1 \leqq i \leqq m, f^{-i}(\gamma)$ and $f^{i}(\gamma)$ are the graphs of a Lipschitz function. Also note that the definition of $L_{z}\left(K_{m}\right)$ is independent of the choice of $z$ such that $\pi(z)=x$, and the definition of $L_{z}^{*}\left(K_{m}\right)$ is independent of $z$ up to translation by $R(t, y)=(t-1, y)$. Note that $L_{z}\left(K_{m}\right)$ $C L_{z}\left(K_{m-1}\right)$.

3.3. "Killends." If for some $m>0$, either $L_{z}^{*}\left(K_{m}\right)=\emptyset$ or $L_{z}\left(K_{m}\right)=\emptyset$, then there can be no rotational invariant circles through $x$. The MacKay and Percival implementation of "killends" tests for $L_{z}^{*}\left(K_{m}\right)=\emptyset$, however it takes very little extra work to test for $L_{z}\left(K_{m}\right)=\emptyset$, and one might as well do this instead. In fact by considering images of the vertical under $F^{n}$, one can show that $L_{z}\left(K_{m}\right)=\emptyset, \forall m>0$, if and only if $L_{z}^{*}\left(K_{m}\right)=\emptyset, \forall m>0$ (Stark, 1986), and hence the two versions are equivalent. In this paper we shall only consider the condition $L_{z}\left(K_{m}\right)=\emptyset$, since this makes the mathematical treatment considerably simpler.

3.4. Lemma. $L_{z}\left(K_{m}\right)$ is bounded $\forall m>0$, that is if $z=\left(t_{0}, y_{0}\right)$ there exists a constant $B$ such that $\left|\gamma(t)-y_{0}\right| \leqq B, \forall t \in \mathbb{R}, \forall \gamma \in L_{z}\left(K_{m}\right)$.

Proof. Since $L_{z}\left(K_{m}\right) \subset L_{z}\left(K_{m-1}\right)$ it is enough to show that $L_{z}\left(K_{1}\right)$ is bounded. Let $V$ be the vertical through $F^{-1}(z)=\left(t_{-1}, y_{-1}\right)$, i.e. $V=\left\{(t, y) \in \mathbb{R}^{2}\right.$ such that $\left.t=t_{-1}\right\}$. Then since $F$ is a twist map, $F(V)$ is the graph of a continuous function $\Psi: \mathbb{R} \rightarrow \mathbb{R}$. By the definition of $K_{1}^{+}$, if $\gamma \in L_{z}\left(K_{m}\right)$ then, $\gamma(t) \geqq \Psi(t), \forall t \leqq t_{0}$ and $\gamma(t) \leqq \Psi(t), \forall t \geqq t_{0}$. So let

$$
B=\sup _{t \in\left[t_{0}-1, t_{0}+1\right]}\left|\Psi(t)-y_{0}\right| .
$$

3.5. Remark. As a consequence of this lemma we only need to evaluate $K_{m}$ on a bounded region in order to prove the non-existence of a rotational invariant circle through a given point.

Another test for non-existence is also required:

3.6. Definition. Let $U \subset \mathbb{T}^{1} \times \mathbb{R}$ be an open set homeomorphic to $\mathbb{T}^{1} \times \mathbb{R}$ such that $\mathbb{T}^{1} \times(-\infty, a] \subset U \subset \mathbb{T}^{1} \times(-\infty, b]$ for some $a, b \in \mathbb{R}$. Then the net flux or Calabi Invariant of a twist map $f$ is $[\operatorname{area}(f U \backslash U)$-area $(U \backslash f U)]$. Since $f$ is areapreserving this is independent of the choice of $U$. It can be thought of as the flux of area across a circle $\{y=$ const $\}$. Clearly if $f$ has a rotational invariant circle then this net flux must be zero, and hence we get:

3.7. Net Flux Test. If the net flux of $f$ is non-zero then $f$ can have no rotational invariant circles.

3.8. Remark. On the other hand there are twist maps with non-zero flux for which $K_{m}^{+}(x)>K_{m}^{-}(x), \forall x \in M, \forall m>0$, e.g. $y^{\prime}=y+1, \theta^{\prime}=\theta+y$ (example due to R. de la Llave, private communication). For such a map cone crossing and "killends" will never prove that there are no rotational invariant circles. We thus need to test for non-zero flux, this can be done by sufficiently precise rigorous numerical integration. Note that in pactice it will usually be obvious whether or not a given twist map has zero net flux (e.g. if $f$ has at least one rotational invariant circle somewhere then it has zero net flux, thus maps of an annulus $\mathbb{T}^{1} \times[0,1]$ always have zero net flux). Furthermore in practical examples (e.g. the standard map) the net flux is usually zero. 


\section{The Finite Computation Conjecture}

Note that all of the criteria described in the previous two sections can be applied to a whole $C^{1}$ ball of twist maps at a time. Indeed this happens naturally in a computer implementation since in that case the map is only determined up to a finite accuracy. MacKay and Percival (1985) then make the following conjecture:

4.1. Finite Computation Conjecture. Let $X$ be a compact subset of $M \times A(M)$, where $A(M)$ is the space of all $C^{1}$ area-preserving twist maps of $M$, such that for all $(x, f) \in X$, the map $f$ has no rotational invariant circle through $x \in M$. Then with a finite amount of computation the combination of the net flux test, cone-crossing and killends will prove that for all $(x, f) \in X$, the map $f$ has no rotational invariant circle through $x$.

4.2. Remark. If any of the net flux test, cone-crossing or killends succeed for some $x \in M, f \in A(M)$, then they also succeed for some open $C^{1}$ ball in $M \times A(M)$ around $(x, f)$. Hence the above conjecture is equivalent to the algorithm based on these criteria being exhaustive.

4.3. Remark. As stated above the conjecture is too vague to admit a mathematical proof. To have some hope of proving it we have to place some reasonable restrictions on the way in which the criteria are implemented: there clearly exist algorithms based on the above criteria which are not exhaustive. A proof of the conjecture will then have two parts:

1) A proof that if $f$ has zero net flux and $L_{z}\left(K_{m}\right) \neq \emptyset, \forall m>0$, i.e. for any $m$ there is a closed curve through $z$ that satisfies the conefield $K_{m}$, then there exists a rotational invariant circle through $z$. Thus if $z$ has no rotational invariant circles through it, either $f$ has non-zero net flux or $L_{z}\left(K_{m}\right)=\emptyset$ for some $m>0$. Hence an idealised criterion which calculates the conefields $K_{m}$ and the net flux exactly will indeed show that there are no invariant circles through $z$.

2) A proof that the restrictions imposed on a practical implementation [using (2.11) rather than (2.7) to calculate the conefields] ensure that it is a sufficiently good approximation of the idealised criterion to also be exhaustive. This essentially amounts to showing that we can calculate the conefields and the net flux to an arbitrarily high precision using rigorous interval arithmetic on a digital computer.

We shall give a proof of 1) below, 2) is rather technical and the details can be found in Stark (1986). Note that the proof gives no upper bound on the amount of computation required, and hence we cannot use this method to prove the existence of invariant circles (which in general is much harder to do than to prove nonexistence). It seems highly unlikely that an upper bound could be given except in special cases, since given a point at which the cones will eventually cross, there is no a priori way of estimating how many iterations of the conefield are required for this to happen.

4.4. Theorem. If $f$ has zero net flux and $L_{z}\left(K_{m}\right) \neq \emptyset, \forall m>0$, then there exists a rotational invariant circle through $z$.

Observe that the converse is trivially true and hence this theorem gives necessary and sufficient conditions for the existence of rotational invariant circles. 
4.5. Definition. Let $R$ be the translation $R(u, v)=(u-1, v)$. If $z=\left(t_{0}, y_{0}\right)$, then for $i \in \mathbb{N}$ define $z_{i}=\left(t_{i}, y_{i}\right)=R^{p(i)} F^{i}(z)$ with $p(i)$ chosen such that $t_{0} \leqq t_{i}<t_{0}+1$.

4.6. Definition. Let

$$
\begin{aligned}
L_{z} & =\bigcap_{m>0} L_{z}\left(K_{m}\right), \\
L & =\bigcup_{z \in \mathbb{R}^{2}} L_{z}, \\
L_{z, n} & =\left\{\gamma \in L_{z} \text { such that } F^{j}(z) \in \text { graph } \gamma, 0 \leqq j \leqq n\right\}, \\
L_{z, \infty} & =\bigcap_{n \geqq 0} L_{z, n} .
\end{aligned}
$$

Clearly if a rotational $f$-invariant circle through $z$ exists it will belong to $L_{z, \infty}$. Our proof of (4.4) will proceed in three stages:

i) $L_{z}\left(K_{m}\right) \neq \emptyset, \forall m>0 \Rightarrow L_{z, 0}=L_{z} \neq \emptyset$.

ii) $\forall n>0, L_{z, n-1} \neq \emptyset \Rightarrow L_{z, n} \neq \emptyset$.

iii) If $L_{z, n} \neq \emptyset, \forall n \in \mathbb{N}$, then there exists a rotational $f$-invariant circle in $L_{z, \infty}$.

Let $C_{\text {per }}(\mathbb{R})$ be the Banach space of continuous functions $\varphi: \mathbb{R} \rightarrow \mathbb{R}$, such that $\varphi(t+1)=\varphi(t), \forall t \in \mathbb{R}$, with the sup norm.

4.7. Lemma. $L_{z}\left(K_{m}\right)$ is closed in $C_{\text {per }}(\mathbb{R}), \forall m>0$.

Proof. If $L_{z}\left(K_{m}\right)=\emptyset$, then it is closed. So suppose not and take a sequence $\gamma_{i} \in L_{z}\left(K_{m}\right)$, with $\gamma_{i} \rightarrow \gamma$, uniformly. Note that $K_{m}^{+}$and $K_{m}^{-}$are continuous as functions $\mathbb{R}^{2} \rightarrow \mathbb{R}$, so let $J^{+}=\sup \left\{K_{m}^{+}(t, \gamma(t)), t \in[0,1]\right\}$ and $J^{-}=\inf \left\{K_{m}^{-}(t, \gamma(t))\right.$, $t \in[0,1]\}$. Recall from $(2.8)$ that $1 / 4>J^{+}$and $J^{-}>-1 / 4$. Let $w_{0}=\left(t_{0}, \gamma\left(t_{0}\right)\right)$ $\in$ graph $\gamma$. Since the $\gamma_{i}$ satisfy $K_{m}$, we have $K_{m}^{+}(x) \geqq K_{m}^{-}(x), \forall x \in \operatorname{graph} \gamma_{i}, \forall i \in \mathbb{N}$. Since $w_{0}$ is in the closure of such points and $K_{m}^{+}$and $K_{m}^{-}$are continuous, we have $K_{m}^{+}\left(w_{0}\right) \geqq K_{m}^{-}\left(w_{0}\right)$ and hence $1 / 4>J^{+} \geqq K_{m}^{+}\left(w_{0}\right) \geqq K_{m}^{-}\left(w_{0}\right) \geqq J^{-}>-1 / 4$. Now given an $\varepsilon>0$ sufficiently small (such that $\varepsilon<1 / 4-J^{+}$and $\varepsilon<J^{-}+1 / 4$ ), $\exists \delta>0$ such that $\forall w \in\left[t_{0}-\delta, t_{0}+\delta\right] \times\left[\gamma\left(t_{0}\right)-\delta, \gamma\left(t_{0}\right)+\delta\right]$, we have $\left|K_{m}^{ \pm}(w)-K_{m}^{ \pm}\left(w_{0}\right)\right|<\varepsilon$. Then on $\left[t_{0}-\delta, t_{0}+\delta\right]$ the $\gamma_{i}$ for $i \geqq N$, some $N \in \mathbb{N}$, have uniform Lipschitz constants $C^{+}$ $=\left[\tan 2 \pi\left(K_{m}^{+}\left(w_{0}\right)+\varepsilon\right)\right] \quad$ and $C^{-}=\left[\tan 2 \pi\left(K_{m}^{-}\left(w_{0}\right)-\varepsilon\right)\right], \quad$ i.e. $\quad C^{-} \leqq\left[\gamma_{i}(t)\right.$ $\left.-\gamma_{i}\left(t^{\prime}\right)\right] /\left(t-t^{\prime}\right) \leqq C^{+}, \forall t, t^{\prime} \in\left[t_{0}-\delta, t_{0}+\delta\right]$. Hence so has $\gamma$. But this holds for all sufficiently small $\varepsilon>0$ and hence $\gamma$ satisfies $K_{m}$ at $w_{0}$.

4.8. Lemma. $L_{z}\left(K_{m}\right)$ is uniformly Lipschitz, i.e. there is a constant $J=J(z, m)$, $0 \leqq J<\infty$ such that $\left|\gamma(t)-\gamma\left(t^{\prime}\right)\right| \leqq J\left|t-t^{\prime}\right|, \forall \gamma \in L_{z}\left(K_{m}\right), \forall t, t^{\prime} \in \mathbb{R}$. In fact since $L_{z}\left(K_{m}\right)$ $C L_{z}\left(K_{m-1}\right)$, we can take $J(z, m)$ independent of $m$.

Proof. If $L_{z}\left(K_{m}\right)=\emptyset$, then the lemma is trivially true. Suppose not. By (3.4), let $A=\mathbb{T}^{1} \times[-B, B]$ be such that $\operatorname{graph} \gamma \subset A, \forall \gamma \in L_{z}\left(K_{m}\right)$. Note that $K_{m}^{ \pm}$are continuous as functions $\mathbb{R}^{2} \rightarrow \mathbb{R}$, so let $J^{+}=\sup \left\{K_{n}^{+}(u), u \in A\right\}$ and $J^{-}=\inf \left\{K_{n}^{-}(u)\right.$, $u \in A\}$. By (2.8), $J^{+}<1 / 4$ and $J^{-}>-1 / 4$. If $\gamma \in L_{z}\left(K_{m}\right)$, then $K_{m}^{+}(x) \geqq K_{m}^{-}(x)$, $\forall x \in \operatorname{graph} \gamma$. Hence $J^{+} \geqq J^{-}$. Let $J=\max \left\{\left|\tan 2 \pi J^{+}\right|,\left|\tan 2 \pi J^{-}\right|\right\}$. Then $0 \leqq J<\infty$ and $\left|\gamma(t)-\gamma\left(t^{\prime}\right)\right|<J\left|t-t^{\prime}\right|, \forall \gamma \in L_{z}\left(K_{m}\right), \forall t, t^{\prime} \in \mathbb{R}$.

4.9. Corollary. $L_{z}\left(K_{m}\right) \neq \emptyset, \forall m>0 \Rightarrow L_{z} \neq \emptyset$. 
Proof. Let $\gamma_{i}, i>0$, be a sequence with $\gamma_{i} \in L_{z}\left(K_{i}\right)$. Since $L_{z}\left(K_{m}\right) \subset L_{z}\left(K_{m-1}\right)$, $\gamma_{i} \in L_{z}\left(K_{j}\right), \forall 1 \leqq j \leqq i$. In particular $\left\{\gamma_{i}, i>0\right\} \subset L_{z}\left(K_{1}\right)$. By (3.4) and (4.8) $L_{z}\left(K_{1}\right)$ is pointwise bounded and equicontinuous. Thus by Ascoli's Theorem (Rudin, 1973), $\gamma_{i}$ has a uniformly convergent subsequence. Let $\gamma$ be the limit of this subsequence. By (4.7), $\gamma \in L_{z}\left(K_{m}\right), \forall m>0$, as required.

4.10. Definition. Since the action of $D F, D F^{-1}$ on angles is order preserving, if $\gamma \in L$, then $\mathrm{F}($ graph $\gamma)$ must also satisfy $K_{m}, \forall m>0$, and hence be in $L$. Thus $F(\operatorname{graph} \gamma)$ is the graph of a Lipschitz function which we denote $F \gamma$. In other words $F \gamma$ is defined by the graph transform:

$$
\operatorname{graph}(F \gamma)=F(\operatorname{graph} \gamma) .
$$

4.11. Remark. If $f$ has zero net flux then $\gamma$ and $F \gamma$ must intersect. More specifically, either $\gamma=F \gamma$ (and so $\gamma$ represents a rotational invariant circle) or $\exists t, t^{\prime} \in[0,1]$ such that $\gamma(t)<(F \gamma)(t)$ and $\gamma\left(t^{\prime}\right)>(F \gamma)\left(t^{\prime}\right)$. If $L_{z, n} \neq \emptyset$, we shall use $F$ to construct a $\gamma \in\left(L_{z, n} \cap L_{F(z), n}\right) \subset L_{z, n+1}$.

4.12. Definition. If $L_{z, n} \neq \emptyset$, define

$$
\begin{array}{ll}
\Gamma_{n}^{+}(t)=\sup _{\gamma \in L_{z, n}} \gamma(t), & t \in \mathbb{R}, \\
\Gamma_{n}^{-}(t)=\inf _{\gamma \in L_{z, n}} \gamma(t), & t \in \mathbb{R}
\end{array}
$$

4.13. Lemma. If for some $n \in \mathbb{N}, L_{z, n} \neq \emptyset$, then $\Gamma_{n}^{+}, \Gamma_{n}^{-} \in L_{z, n}$.

Proof. By (4.8), let $J$ be a Lipschitz constant for $L_{z}\left(K_{1}\right)$. Since $L_{z, n} \subset L_{z}\left(K_{1}\right)$, $\left|\gamma(t)-\gamma\left(t^{\prime}\right)\right| \leqq J\left|t-t^{\prime}\right|, \forall \gamma \in L_{z, n}, \forall t, t^{\prime} \in \mathbb{R}$. Hence the same holds for $\Gamma_{n}^{ \pm}$. For any $i \in \mathbb{N}$, choose $N_{i}$ such that $N_{i}>2 i J$. For $k=0, \ldots, N_{i}-1$, choose $\gamma_{i, k} \in L_{z, n}$ such that $\left|\Gamma_{n}^{ \pm}\left(k / N_{i}\right)-\gamma_{i, k}\left(k / N_{i}\right)\right|<1 / 2 i$, (Fig. 4). Define $\gamma_{i}$ by $\gamma_{i}(t)=\max$ or $\min \left\{\gamma_{i, k}(t)\right.$, $\left.k=0, \ldots, N_{i}-1\right\}$ respectively. Note that $\gamma_{i} \in L_{z, n}$. Then $\left|\Gamma_{n}^{ \pm}(t)-\gamma_{i}(t)\right|<1 / i, \forall t \in \mathbb{R}$, $\forall i \in \mathbb{N}$, i.e. $\gamma_{i} \rightarrow \Gamma_{n}^{ \pm}$uniformly and thus by (4.7), $\Gamma_{n}^{ \pm} \in L_{z}$. But by definition $F^{j}(z) \in \operatorname{graph} \Gamma_{n}^{ \pm}, 0 \leqq j \leqq n$, and thus $\Gamma_{n}^{ \pm} \in L_{z, n}$ as required.

4.14. Lemma. Suppose $L_{z, n} \neq \emptyset$ for some $n \in \mathbb{N}$ and $z=\left(t_{0}, y_{0}\right)$. Then:

$$
\left(F \Gamma_{n}^{-}\right)\left(t_{0}\right) \leqq y_{0} \leqq\left(F \Gamma_{n}^{+}\right)\left(t_{0}\right) .
$$

Fig. 4

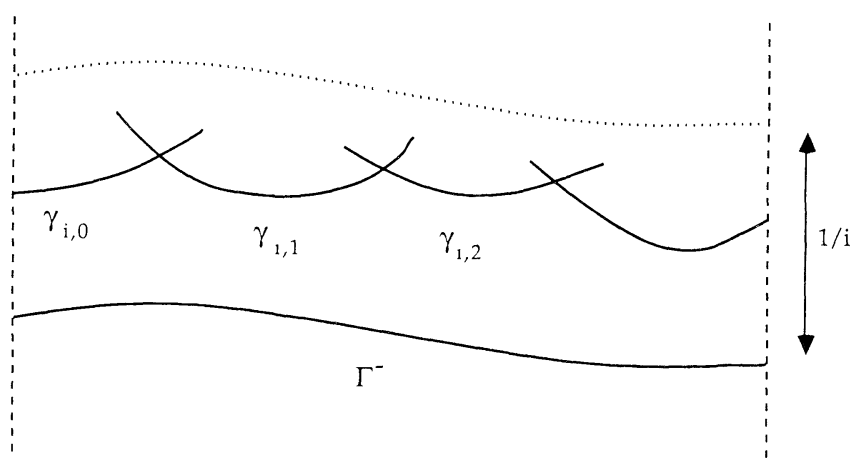




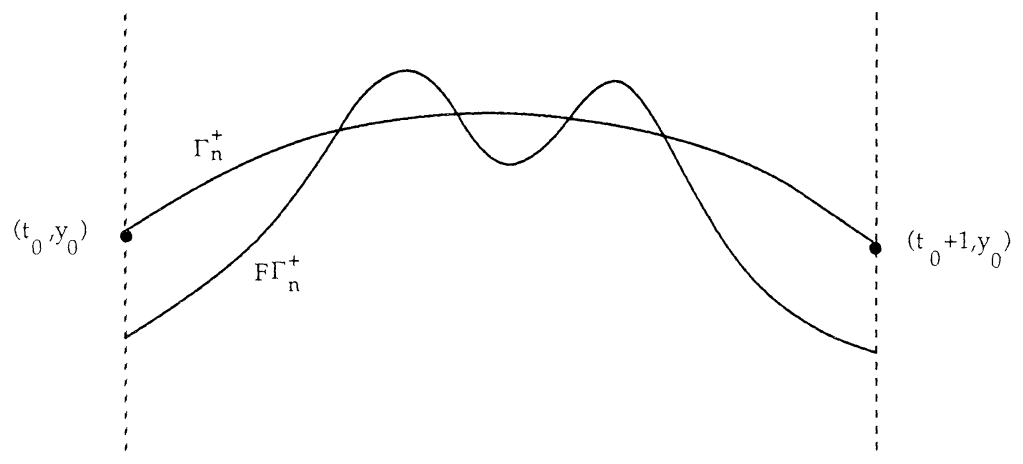

Fig. 5

Proof. We shall prove $y_{0} \leqq\left(F \Gamma_{n}^{+}\right)\left(t_{0}\right)$; the other half of the inequality is proved similarly. So suppose $\Gamma_{n}^{+}\left(t_{0}\right)=y_{0}>\left(F \Gamma_{n}^{+}\right)\left(t_{0}\right)$, (Fig. 5). By zero net flux $\exists t^{\prime} \in\left[t_{0}, t_{0}+1\right]$ such that $\left(F \Gamma_{n}^{+}\right)\left(t^{\prime}\right)>\Gamma_{n}^{+}\left(t^{\prime}\right)$. Define $\psi$ by:

$$
\psi(t)=\max \left\{\Gamma_{n}^{+}(t),\left(F \Gamma_{n}^{+}\right)(t)\right\} \quad \forall t \in \mathbb{R} .
$$

Then $\psi \in L$. Since $\Gamma_{n}^{+}\left(t_{0}\right)>\left(F \Gamma_{n}^{+}\right)\left(t_{0}\right), \quad \psi\left(t_{0}\right)=y_{0}, \quad$ so $z \in \operatorname{graph} \psi$. Also $F^{j}(z) \in \operatorname{graph} \Gamma_{n}^{+}, \forall 0 \leqq j \leqq n$, and hence $F^{j}(z) \in \operatorname{graph}\left(F \Gamma_{n}^{+}\right), \forall 1 \leqq j \leqq n+1$. Thus $\Gamma_{n}^{+}\left(t_{j}\right)=y_{j}=\left(F \Gamma_{n}^{+}\right)\left(t_{j}\right), \forall 1 \leqq j \leqq n$, and so $\psi\left(t_{j}\right)=y_{j}, \forall 1 \leqq j \leqq n$. Hence $F^{j}(z) \in \operatorname{graph} \psi$, $\forall 1 \leqq j \leqq n$. So $\psi \in L_{z, n}$. But $\left(F \Gamma_{n}^{+}\right)\left(t^{\prime}\right)>\Gamma_{n}^{+}\left(t^{\prime}\right)$, and thus $\psi\left(t^{\prime}\right)>\Gamma_{n}^{+}\left(t^{\prime}\right)$, which contradicts the definition of $\Gamma_{n}^{+}$.

4.15. Lemma. Suppose that $L_{z, n} \neq \emptyset$ for some $n \in \mathbb{N}$ and $z=\left(t_{0}, y_{0}\right)$. Then (see Fig. 6 , drawn for the case $n=0$ ):

$$
\begin{aligned}
& \left(F \Gamma_{n}^{+}\right)(t) \geqq \Gamma_{n}^{-}(t) \quad \forall t \in \mathbb{R}, \\
& \left(F \Gamma_{n}^{-}\right)(t) \leqq \Gamma_{n}^{+}(t) \quad \forall t \in \mathbb{R} .
\end{aligned}
$$

Proof. We only prove the first inequality; the proof of the other is similar. Define $\varphi$ by

$$
\varphi(t)=\min \left\{\Gamma_{n}^{-}(\mathrm{t}),\left(\mathrm{F} \Gamma_{n}^{+}\right)(\mathrm{t})\right\} \quad \forall t \in \mathbb{R} .
$$

Fig. 6

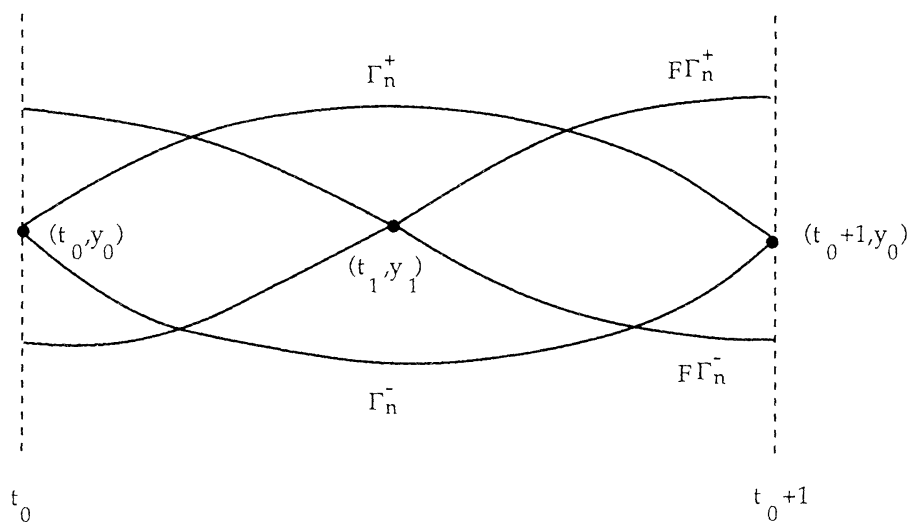


Then $\varphi \in L$. By Lemma 4.14, $\Gamma_{n}^{-}\left(t_{0}\right)=y_{0} \leqq\left(F \Gamma_{n}^{+}\right)\left(t_{0}\right)$, hence $\varphi\left(t_{0}\right)=y_{0}$. So $z \in \operatorname{graph} \varphi$, i.e. $\varphi \in L_{z}$. As in the proof of Lemma 4.14 we have $F^{j}(z) \in \operatorname{graph} \varphi$, $\forall 1 \leqq j \leqq n$. So $\varphi \in L_{z, n}$, which by the definition of $\Gamma_{n}^{-}$, gives $\varphi(t) \geqq \Gamma_{n}^{-}(t), \forall t \in \mathbb{R}$. But $\left(F \Gamma_{n}^{+}\right)(t) \geqq \varphi(t), \forall t \in \mathbb{R}$, and hence $\left(F \Gamma_{n}^{+}\right)(t) \geqq \Gamma_{n}^{-}(t), \forall t \in \mathbb{R}$, as required.

4.16. Lemma. If $L_{z} \neq \emptyset$ then $L_{z, n} \neq \emptyset, \forall n \in \mathbb{N}$.

Proof. By induction $L_{z, 0}=L_{z} \neq \emptyset$. So now suppose that for some $n \in \mathbb{N}, L_{z, n} \neq \emptyset$. Define $\eta$ by (see Fig. 6, drawn for the case $n=0$ )

$$
\eta(t)=\min \left\{\Gamma_{n}^{+}(t),\left(F \Gamma_{n}^{+}\right)(t)\right\} \quad \forall t \in \mathbb{R} .
$$

Then $\eta \in L$. By Lemma 4.14, $\Gamma_{n}^{+}\left(t_{0}\right) \leqq\left(F \Gamma_{n}^{+}\right)\left(t_{0}\right)$, hence $\eta\left(t_{0}\right)=y_{0}$. As in the proof of Lemma 4.14 we have $F^{j}(z) \in$ graph $\eta, \forall 1 \leqq j \leqq n$, and so $\eta \in L_{z, n}$. By Lemma 4.15, $\left(F \Gamma_{n}^{-}\right)\left(t_{n+1}\right) \leqq \Gamma_{n}^{+}\left(t_{n+1}\right)$. But $\left(F \Gamma_{n}^{+}\right)\left(t_{n+1}\right)=y_{n+1}=\left(F \Gamma_{n}^{-}\right)\left(t_{n+1}\right)$, so $\left(F \Gamma_{n}^{+}\right)\left(t_{n+1}\right)$ $\leqq \Gamma_{n}^{+}\left(t_{n+1}\right)$. Thus $\eta\left(t_{n+1}\right)=y_{n+1}$, and so $F^{n+1}(z) \in \operatorname{graph} \eta$. Hence $\eta \in L_{z, n+1}$ as required.

Proof of Theorem 4.4. By above $\Gamma_{n}^{+}$exists $\forall n \in \mathbb{N}$. $\Gamma_{n}^{+} \in L_{z}, \forall n \in \mathbb{N}$, and by (3.4) and (4.8), $L_{z}$ is pointwise bounded and equicontinuous. Hence by Ascoli's theorem (Rudin, 1974) $\Gamma_{n}^{+}$has a uniformly convergent subsequence. Let $\Gamma$ be the limit of this subsequence. Then $\Gamma \in L_{z}$ by (4.7). Since $L_{z, n+1} C L_{z, n}$, we have $F^{n}(z) \in \operatorname{graph} \Gamma$, $\forall n \in \mathbb{N}$ and hence $\Gamma \in L_{z, \infty}$. It remains to show that $F \Gamma=\Gamma$. Note that $\Gamma(t) \geqq \gamma(t)$, $\forall t \in \mathbb{R}, \forall \gamma \in L_{z, \infty}$. Now $\left(F^{-1} \Gamma\right) \in L$ and $F^{n}(x) \in \operatorname{graph}\left(F^{-1} \Gamma\right), \forall n \in \mathbb{N}$, hence $\left(F^{-1} \Gamma\right) \in L_{z, \infty}$. So $\Gamma(t) \geqq\left(F^{-1} \Gamma\right)(t), \forall t \in \mathbb{R}$. But $F^{-1}$ has zero net flux, hence if $\exists t^{\prime} \in \mathbb{R}$ such that $\Gamma\left(t^{\prime}\right)>\left(F^{-1} \Gamma\right)\left(t^{\prime}\right)$ then $\exists t^{\prime \prime} \in \mathbb{R}$ such that $\Gamma\left(t^{\prime \prime}\right)<\left(F^{-1} \Gamma\right)\left(t^{\prime \prime}\right)$. Hence $\Gamma(t)=\left(F^{-1} \Gamma\right)(t), \forall t \in \mathbb{R}$, and so $F \Gamma=\Gamma$ as required.

Acknowledgements. I am indebted to R. S. MacKay for his many helpful suggestions and constant encouragement, and in particular for explaining the criteria discussed in this paper. I would also like to thank Professor E. C. Zeeman, K. Grosse-Brauckmann, and R. Sims for useful conversations and Prof. I. C. Percival for a number of helpful comments on an earlier draft of this paper. Lastly I am grateful to the UK Science and Engineering Research Council for financial support.

\section{References}

Aubry, S.: The twist map, the extended Frenkel-Kontorova model and the Devil's staircase. Physica 7 D, 240-258 (1983)

Aubry, S., Le Daeron, P.Y.: The discrete Frenkel-Kontorova model and its extensions. I. Exact results for the ground states. Physica $8 \mathrm{D}, 381-422$ (1983)

Birkhoff, G.D.: Surface transformations and their dynamical applications. Acta Math. 43, 1-119 (1920)

Birkhoff, G.D.: Sur quelques courbes fermees remarquables. Bull. Soc. Math. Fr. 60, 1 (1932)

Boyland, P.L., Hall, G.R.: Invariant circles and the order structure of periodic orbits in monotone twist maps. Topology 26, 21-36 (1987)

Chirikov, B.V.: A universal instability of many dimensional oscillator systems. Phys. Rep. $\mathbf{5 2}$, 263-379 (1979)

Greene, J.M.: A method for determining a stochastic transition. J. Math. Phys. 20, 1183-1201 (1979)

Herman, M.R.: Sur les courbes invariantes par les diffeomorphismes de l'anneau, Vol. 1. Asterisque 103-104 (1983) 
Katok, A.: Some remarks on Birkhoff and Mather twist map theorems. Erg. Theory Dyn. Sys. 2, 185-194 (1982)

Katok, A.: Periodic and quasi-periodic orbits for twist maps. In: Dynamical systems and chaos, proceedings, Sitges 1982. Garrido, L. (ed.). Berlin, Heidelberg, New York: Springer 1983

Leage, I., MacKay, R.S.: Badly ordered periodic orbits of the standard map. Phys. Lett. A 118, 274-278 (1986)

MacKay, R.S.: Renormalisation in area-preserving maps. PhD Thesis, Princeton 1982

MacKay, R.S.: A renormalisation approach to invariant circles in area-preserving maps. Physica $7 \mathrm{D}, 283-300(1983)$

MacKay, R.S., Meiss, J.D., Percival, I.C.: Transport in Hamiltonian systems. Physica 13 D, 55-81 (1984)

MacKay, R.S., Percival, I.C.: Converse KAM: Theory and practice. Commun. Math. Phys. 98, 469-512 (1985)

MacKay, R.S., Stark, J.: Lectures on orbits of minimal action for area-preserving maps. University of Warwick preprint (1985)

Mather, J.N.: Non-existence of invariant circles. Erg. Theory Dyn. Sys. 4, 301-311 (1984)

Mather, J.N.: A criterion for the non-existence of invariant circles. Math. Publ. IHES. 63, 153-204 (1986)

Newman, R.A.P.C., Percival, I.C.: Definite paths and upper bounds on regular regions of velocity phase space. Physica 6 D, 249-259 (1983)

Rudin, W.: Functional analysis. Appendix A. New York: Tata McGraw-Hill 1973

Stark, J.: On invariant circles for area-preserving maps. PhD. Thesis, University of Warwick 1986

Communicated by $\mathbf{J}$. Mather

Received December 3, 1986; in revised form November 16, 1987 
